\title{
TIMELINESS FOR DYNAMIC SOURCE SELECTION IN SITUATED PUBLIC DISPLAYS
}

\author{
F. Reinaldo Ribeiro \\ Departamento de Informática, Escola Superior de Tecnologia, Castelo Branco, Portugal \\ fribeiro@est.ipcb.pt \\ Rui José \\ Departamento de Sistemas de Informação, Universidade do Minho, Guimarães, Portugal \\ rui@dsi.uminho.pt
}

Keywords: Dynamic Sources, Information Integration, Timeliness, Public Displays, Situated Displays, Social Information Systems, Web Information Filtering and Retrieval, Web 2.0.

Abstract: Dynamic sources, which make regularly updated data available for use by other applications, are increasingly a key enabling feature of the web. They are extensively used in all sorts of social media applications where they are re-combined in multiple ways to generate new aggregate services. Public situated displays are an emergent area where dynamic sources can also play a key role in providing situated and frequently updated content. However, the specificities of public displays raise the need for automated selection of the most relevant sources to present. This study addresses relevance from the perspective of timeliness. We propose a timeliness model that supports the most common types of dynamic source. To validate that model, we set an experiment with a public display exhibiting content from dynamic sources and receiving from users feedback on its timeliness. The results from this experiment suggest a reasonable match between our model and the users' perspectives on timeliness. The results also show that the model is able to make comparative calculations of timeliness for different types of dynamic source. These results enable us to conclude that timeliness functions may help to significantly increase the relevance of content automatically selected from dynamic sources.

\section{INTRODUCTION}

A key enabling feature of the Web in the social software era is the integration of multiple data sources into combined services that exhibit an aggregate view that is constantly being updated from the original sources. This model is extensively used in social media applications and is also at the core of the mashup concept, in which information from various sources is recombined to form new applications. In this paper we will use the term Dynamic Source to refer to these information sources that make regularly updated data available for use by other applications and look in particular at how they can be leveraged for the generation of content for digital situated displays.

Public situated displays are an emergent area where dynamic sources can play a key role in providing situated and frequently updated content. However, the common scenario for interaction with public displays is very different from the traditional web scenarios and raises specific challenges that may limit the applicability of dynamic sources as content generators. The problem with dynamic sources is that, precisely because they are dynamic, the relevance of the respective information is likely to face considerable oscillations. Any particular source may, at some point, be producing content that is timely while at some other point may have nothing to show or its content may be strongly deprecated. For example, a feed from a blog with many recent messages on some hot topic may be very relevant when the new messages are being posted and then quickly become outdated when the posting activity stops.

In a traditional web scenario these variations in the relevance of the sources are not a major concern. The navigation experience gives people full control over which information to access and many cues on 
which information to select. Multiple data items from various sources are typically presented in the form of short summaries with links for further details, and people can easily evaluate which ones may be of interest and navigate accordingly. On the contrary, in a public display, the interaction model is essentially a push model, in which the system makes most of the decisions on what is going to be presented next. People are very limited in their ability to influence the display decisions, not only for the technical considerations resulting from the lack of a mouse and keyboard, but essentially due to the fact that the display is public and shared. Furthermore, given that people will not normally have the possibility to request for further details, all the content is presented. As a result, there is a high probability that at any moment the display will be showing deprecated or otherwise irrelevant information.

In this work, we explore an alternative model that basically consists in maintaining a potentially large pool of possible sources and selecting for presentation only those that are currently more relevant. In general, the relevance of a particular resource is an indication of the pertinence of that resource to the current needs of the users, but in this work we are only concerned with the time dimension, i.e. evaluating how timely the information is.

This notion of timeliness is of an obvious importance in setting the relevance for any type of source, but different sources will handle the effect of time differently. For most sources, the relevance measure should guarantee that the information has not lost its value since publication, but in some cases, a higher relevance may be associated with a particular point in time, e.g. the day of an event, and not necessarily decay as time goes by.

The objective of this work is to develop a set of methods for optimizing the timeliness of content from dynamic sources selected for presentation at public displays. This broad research goal embraces the following set of research objectives: to understand the key criteria for evaluating the timeliness of content across several types of dynamic source; to propose and validate a model for timeliness; to uncover any elements that may affect people's perception of timeliness.

To pursue these goals, we started by analyzing time-related meta-data from a large number of real sources. Based on that analysis, we propose two timeliness formulas for two common types of source, those based on a publication date and those based on a planned event date. To support the evaluation of that model we created a public display system where date items were scheduled using those formulas and asked people to classify the timeliness of what was being presented. This was complemented with another experiment designed to investigate the fairness of the model when comparing the timeliness of sources with different time criteria. Results show a clear relation between timeliness as determined from our formulas and timeliness as perceived by people.

\section{RELATED WORK}

Research on situated public displays has received considerable attention recently, with many projects addressing the issues of how to enable information access and share, and enhance collaboration within organizations or communal spaces (Russell and Sue 2002). The BlueScreeen project (Payne, David et al. 2006) selects and displays adverts in response to users detected in the audience. It utilizes Bluetooth-enable devices as proxies for identifying users and utilizes history information of past users' exposure to certain sets of adverts. Advertisements are preferentially shown to those users that have not seen them yet. Muller (Muller, Kruger et al. 2007) describes a mechanism to adapt advertisements on digital signage to the interests of the audience. Here, each advertisement has a set of keywords and the system keeps a history of all advertisements a user was interested in. Groupcast (McCarthy, Costa et al. 2001) is a display that respond to the local audience within a corporate environment to display media contents. It explores user identification and their profiles to identify common areas of interest.

This work also builds on previous work in recommendation systems and retrieval models for feed search (Bihun, Goldman et al. 2007; Seo and Croft 2007; Arguello, Elsas et al. 2008). A key distinguishing characteristic is the different set of assumptions of the specific problem domain. Previous work has address the issue mostly as an information retrieval problem, where the starting point is some type of search phrase, user profile, or interaction history that enables relevance of new items to be determined by the similarity to the search query. Our goal is not to achieve a match between potential sources and any representation of users' interests, simply because we do not have any such representation. In this work, we focus on the evaluation of relevance in a way that is inherent to the source and independent of the presentation context. More specifically, we define our problem as a problem of selecting from a fixed set of sources the items that are currently more timely to present. 


\section{DYNAMIC SOURCES AND TIME}

A dynamic source is specified by the indication of the source that produces it and by a collection of query parameters, such as search keys or constraints that determine the dataset to be produced. This specification, frequently in the form of a URL, represents a formal statement of a particular information need. As a result of an access to the resource, a dataset is produced that is normally composed by multiple data items. Depending on the type of source, these data items may be text, images, videos or any other media type, and they may have their own individual metadata. The resource is expected to be regularly updated and regularly consumed, using methods such as dedicated APIs or XML feeds. As a result, the set of data items returned by the source may vary in subsequent requests for the same resource, and each individual item may itself be updated.

A key part of this work is a model for the timeliness of the data items obtained from dynamic sources. A high level goal for that model is to achieve a reasonable match with common sense notions of timeliness. Additionally, such model should also address the following requirements:

- R1: Leverage on the time-related metadata that is effectively available in the data items

- R2: Address the time-related specificities of the various types of data items, while enabling their comparison in terms of timeliness.

- R3: Be optimized for automated scheduling in which the timeliest content is cyclically selected from a pool with potential sources.

\subsection{Time Related Meta-data}

To identify the possible criteria for calculating timeliness, and particularly to understand the implication of the requirement $\mathrm{R} 1$, we started this work with an analysis of time-related meta-data across a varied and representative sample of dynamic sources, including news feeds, blogs, event announcements and queries to social software web sites. The objective was to study the key characteristics of a representative set of dynamic sources in order to identify the main criteria for calculating timeliness taking into account the data and metadata produced by the various types of resource. Through a period of 3 weeks, we have collected time-related parameters from 117 sources of various types. We have analyzed the time-related data that was actually available for those types and its update frequency. Based on that analysis, we identified three main groups of sources: information items with publication date, event-related items with event date, and content shared on social software web sites. The first two are clearly distinct in the semantics of their time-related meta-data, as we will describe next. The social software web sites were harder to aggregate because each site has its own time semantics, which greatly undermines any attempt of using a common model. We thus chose not to address the sources from that particular group and focus our study only on the first two groups.

\subsection{A Timeliness Model}

The next phase in our work was the definition of a timeliness model for each of the two groups identified. We chose to study the timeliness of individual items rather than the timeliness of dynamic sources, because the data items on any particular source will typically exhibit very distinct time-related parameters that would distort the selection process.

In the case of information items with publication date, timeliness is essentially determined by the time elapsed since the time of publication. However the decay factor associated may vary considerably across different types of sources, which leads to introduce a decay parameter that defines the decay of the information with time (Equation 1).

$$
T_{i}=e^{-\frac{t-P D_{i}}{K_{i}}}
$$

Where:

- $T_{i}$ : the timeliness of the item $i$

- $\quad P D_{i:}$ the publish date of item $i$;

- $t$ : the actual time;

- $K_{i}$ : decay level of the source for item $i$

In the case of event-related items with event date, timeliness is essentially tied to the date of the event, steadily growing as the event approaches and then dropping abruptly as the event comes to an end (Equation 2).

$T_{i}=\left(\frac{0,5}{1+e^{\frac{l_{1 i}+h_{1 i}-2 t}{h_{1 i}-l_{1 i}}}}+\frac{0,5}{1+e^{\frac{l_{2 i}+h_{2 i}-2 t}{h_{2 i}-l_{2 i}}}}\right) \times h\left(S T_{i}-t\right)$

Where:

- $\quad l_{1}, h_{1}, l_{2}$ and $h_{2}$ represent change times for the timeliness function (defined as amount of time since this point to event start time);

- $S T_{i}$ : the event start time;

- $t$; the actual time;

- $h(t)$ :Heaviside step function. 


\section{EXPERIMENT}

To validate the previous models and also to uncover any meaningful user perspectives on timeliness, we set an experiment with a public display showing content from dynamic sources and receiving feedback from users on the timeliness of the content. For this trial, we developed a display system that used different scheduling algorithms to select the next item to display and we then asked users what they thought about the timeliness of what was being presented on the display. This was complemented with an evaluation of the fairness of the algorithms when choosing between multiple source categories.

\subsection{Timely Display System}

We have developed a timely display system that collects data from a pool of predefined dynamic sources, selects the timeliest items and displays the respective content on a public display. As represented in Figure 1, the key input for the system is the set of dynamic sources considered for our study. Those sources are organized in categories, with the items in the same category sharing the same timeliness formula and respective parameters.

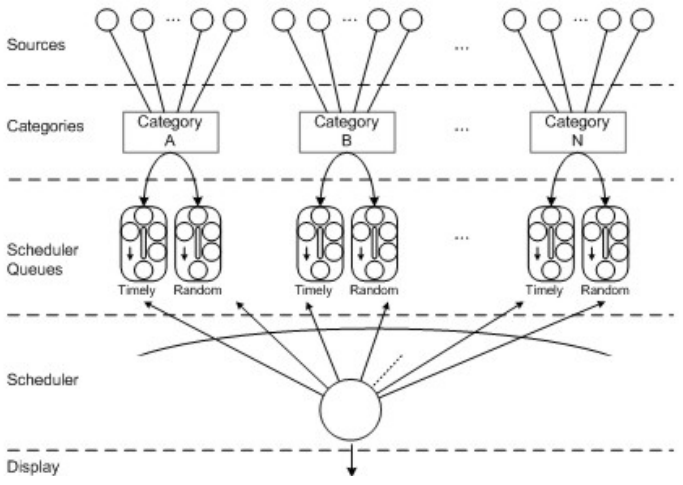

Figure 1: Timely display system.

Two different scheduling queues were created for each category: a timely queue and a random queue. The timely queue contains the 15 items, from all the items in all the sources in the respective category that ranked higher according to the applied timeliness formula (see table 1). The random queue also contains 15 items, but randomly selected from the same category. The timeliness value is regularly updated to reflect not only the passage of time, but also the new items being produced by the sources.

The set of queues from the various categories is the input for the scheduler, which must select the next item that is effectively going to be presented. Each time the scheduler needs to select a new item, it picks an item from one of the queues. In this experiment with users, this selection was made at random, in order to help distribute the number of schedules between all categories including the random ones. The selection within each queue follows a simple round-robin algorithm. Information was displayed as represented in Figure 2.

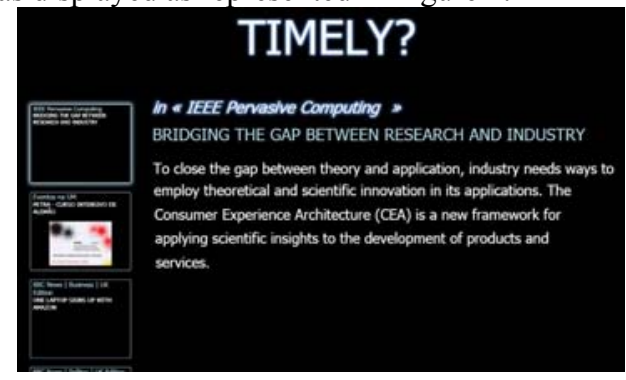

Figure 2: Situated display screenshot.

At the left we have the next items to be presented. The main display area displays the information of the currently selected item, but does not include any reference to time related meta-data. Every 25 seconds the display information is updated with a new item being selected for presentation.

For the purpose of this study, we selected a total of 117 dynamic sources of general interest for our target community. Those sources were grouped according to the nature of their source into the five categories described in Table 1.

Table 1: Categories and parameters (ns:number of sources; $t f$ : timeliness function; $p$ : parameters; $t i$ : total items)

\begin{tabular}{|c|c|c|c|c|}
\hline Category & $n s$ & $t f$ & $p$ & $t i$ \\
\hline News & 38 & Eq. 1 & $\mathrm{~K}=24$ & $\cong 900$ \\
\hline Magazines W. & 46 & Eq. 1 & $\mathrm{~K}=48$ & $\cong 900$ \\
\hline Blogs & 22 & Eq. 1 & $\mathrm{~K}=48$ & $\cong 400$ \\
\hline Announcements & 10 & Eq. 1 & $\mathrm{~K}=48$ & $\cong 250$ \\
\hline Events & 1 & Eq. 2 & $\begin{array}{c}1_{1}=120 ; \mathrm{h}_{1}=96 ; \\
1_{2}=36 ; \mathrm{h}_{2}=24 ;\end{array}$ & $\cong 10$ \\
\hline
\end{tabular}

News and headlines are frequently updated sources (e.g., from TVs, newspapers). Blogs includes content from blogs (usually opinions and/or comments). Magazines and websites represent news from magazines, websites and similar sources on specific type of contents. Announcements category includes contents like classified announcements and advertisements. Finally, events represents sources for which timeliness is strictly connected to the event start date. The total number of items in each category (column ti) is just an indicative value, as this number is always changing due to the dynamic nature of the content sources. Parameters were defined according the nature of the content and our own perception of how their timeliness could evolve. 


\subsection{The Display Setting}

The experiment took place at a reception hall. The setting is composed by two different displays: the Information Display and the Feedback Display. The Information Display shows the items that were selected for presentation. The Feedback display is a small touch screen display that is used to collect users' opinions about the timeliness of what is being presented on the Information display. The display poses to the users the question "What is your opinion about the timeliness of the content that is presented on the display?" and users are able to select between four possible answers (2 if Very Timely; 1 if Timely; 0 if Not Timely and -1 if No Opinion). Each user response is associated to the content that is currently on the situated display and is stored in a database. To prevent the same user from voting multiple consecutive times a delay of five seconds was introduced between feedbacks.

Every time a new item is scheduled, the system registers the schedule start time; schedule end time; scheduler queue; item source; item title; item link; item publication date and event start date (if content is an event). Every time a user gives feedback, the system registers the user opinion on content timeliness and associates it with the displayed item.

\section{RESULTS AND DISCUSSION}

During the 3-weeks of our experiment the display made 33823 schedule decisions corresponding to 8577 distinct items belonging to 102 sources (no items were selected from 15 sources). For the same period we collected a total of 669 timeliness classifications. To improve the quality of the data we eliminated classifications made very close to the moment of transition between items (for which there was some ambiguity on the association) and at night (for which there were very few people). In the end, our analysis was based on 320 valid classifications referring to 239 distinct items from 67 distinct sources.

\subsection{Timeliness Perspectives}

The first goal of our data analysis was to identify how the timeliness of the items selected using our timeliness model had been perceived differently, when compared with the perception of timeliness in the randomly selected items. Figure 3 shows the "mean" value of user classifications for timely and random queue for each category.

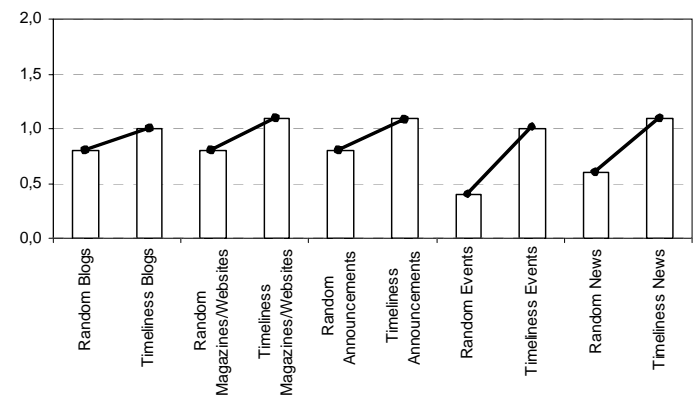

Figure 3: Comparative statistical analysis.

When comparing the two queues in each category a considerable improvement can be observed for all categories. The least successful category is blogs with a $25 \%$ improvement.

The graphs in Figure 4 display the timeliness function for new along with the respective timeliness classifications submitted by people. The horizontal axis represents time in hours since publication or until the event date.

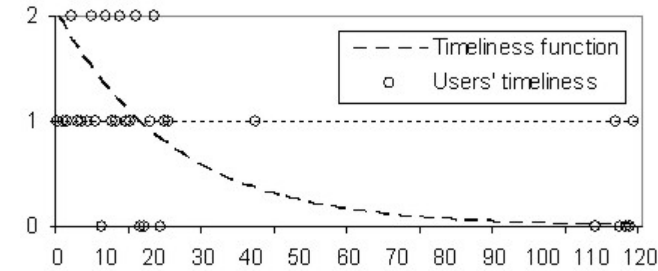

Figure 4: News:Timeliness functions vs users' evaluation.

The existence of randomly selected items has allowed us to obtain classifications for items that were ranking low in timeliness and despite a relative scattering on users' evaluations, there seems to be a clear match between our formula and the classifications made by people.

\subsection{Fairness Between Categories}

A complimentary experiment was made to assess the fairness of the timely algorithm when competitively selecting items from multiple categories. We used the same sources and categories as the basic input, but this time, for each category, we considered a single queue based on our timely algorithm. The selection of the data items to be scheduled was made by selecting from all those queues the 30 most timely items, regardless of their category. This forced the data items of all the categories to compete among each other for scheduler selection.

For a period of 6 days, we registered the schedules made and the number of items available in 
each category. The results are presented in Table 2 and are divided into two parts. The first corresponds to the period between 8 am and $8 \mathrm{pm}$, and the second corresponds to the entire day period.

Table 2: Results for fairness between categories.

\begin{tabular}{|c|c|c|c|c|c|}
\hline \multirow{3}{*}{ 8am-8pm } & \multirow{2}{*}{\multicolumn{2}{|c|}{$\begin{array}{c}\text { Available } \\
\text { items (1) }\end{array}$}} & \multicolumn{3}{|c|}{ Display (2) } \\
\hline & & & \multirow{2}{*}{\begin{tabular}{|c|} 
Total \\
schedules
\end{tabular}} & \multirow{2}{*}{$\begin{array}{c}\% \text { of air } \\
\text { time }\end{array}$} & \multirow{2}{*}{$\begin{array}{l}\% \text { distinct } \\
\text { items }\end{array}$} \\
\hline & start & $\Delta$ new & & & \\
\hline News & 703 & 3166 & 4541 & $53,8 \%$ & $21,7 \%$ \\
\hline$B \operatorname{logs}$ & 290 & 112 & 1099 & $13,0 \%$ & $22,1 \%$ \\
\hline Announc. & 98 & 13 & 8 & $0,1 \%$ & $1,8 \%$ \\
\hline Mag \& W & 766 & 427 & 2659 & $31,5 \%$ & $21,7 \%$ \\
\hline Events & 10 & 7 & 132 & $1,6 \%$ & $17,7 \%$ \\
\hline \multicolumn{6}{|l|}{ All Day } \\
\hline News & 703 & 3428 & 9705 & $48,4 \%$ & $26,8 \%$ \\
\hline$B \log s$ & 290 & 112 & 3221 & $16,1 \%$ & $23,1 \%$ \\
\hline Announc. & 98 & 13 & 40 & $0,2 \%$ & $2,7 \%$ \\
\hline Mag \& W & 766 & 451 & 6752 & $33,7 \%$ & $29,3 \%$ \\
\hline Events & 10 & 7 & 320 & $1,6 \%$ & $35,3 \%$ \\
\hline
\end{tabular}

(1) Number of items at the start of the experiment and number of new items published during the experiment.

(2) Total number of schedules, $\%$ of the total number of schedules and $\%$ of items that were scheduled.

When comparing the fairness between categories, we can observe that some categories are able to gain many more schedules that others. This is in part due to their natural dynamic, but still it is an indicator that some of the parameters in the formulas may have to be fine tuned to increase fairness. Another interesting effect is the existence of differences between the daily period and all day period. This were due to the nature of some sources (e.g. usually blogs are updated out of the day period) and also because of their origin (many of the magazines were from sources with different time zones from our one). The relatively high number of schedules on events is justified because there were four events occurring during this experiment. We can also observe that only part of the items were ever displayed, e.g. $21,7 \%$ for news during the day period. This was a natural consequence of the fact that we had a much higher number of potential data items than time to present them all.

\section{CONCLUSIONS}

This study has investigated how the notion of timeliness can be added to dynamic sources and contributes to improve the relevance of data items selected for presentation in public displays. We have proposed a formula for modeling the timeliness of various types of dynamic sources that builds on time related meta-data effectively available on common sources and is simple to calculate.

The results of the study suggest a reasonable match between our concept of timeliness and the concept as perceived by users. Therefore the introduction of timeliness as a criterion for item selection is expected to have an impact on the perceived relevance of the data presented in public displays. Evaluation of fairness has shown that there are multiple factors that must be considered to ensure a balanced selection among multiple categories or even among the various sources in the same category, including different time zones and different dynamics in the generation of new items. A change in the model suggested by those results is the introduction in the formula of an initial period with no decay to attenuate the effect of different time zones and support a better match with daily rhythms. A more in-depth study of those effects and how to handle them is part of future work in this topic.

\section{ACKNOWLEDGEMENTS}

The first author was supported by a FCT scholarship (SFRH/BD/31292/2006).

\section{REFERENCES}

Arguello, J., J. L. Elsas, et al., 2008. Document Representation and Query Expansion Models for Blog Recommendation. Int. Conf. on Weblogs and Social Media, Seatle.

Bihun, A., J. Goldman, et al., 2007. Ranking blog documents. US Patent \& Trademark Office.

McCarthy, J. F., T. J. Costa, et al., 2001. UniCast, OutCast \& GroupCast: Three Steps toward Ubiquitous Peripheral Displays. Int. Conf. on Ubiquitous Computing, Springer-Verlag.

Muller, J., A. Kruger, et al., 2007. Maximizing the Utility of Situated Public Displays. Adjunct Proceedings of User Modeling, Corfu.

Payne, T., E. David, et al., 2006. Auction Mechanisms for Efficient Advertisement Selection on Public Displays. European Conference on Artificial Intelligence.

Russell, D. M. and A. Sue, 2002. Using Large Public Interactive Displays for Collaboration. W. on Collaboration with Interactive Walls and Tables.

Seo, J. and W. B. Croft, 2007. UMass at TREC 2007 Blog Distillation Task. Text Retrieval Conference. 